\title{
Propiedades psicométricas del Work Preference Inventory (WPI) en una muestra de adultos jóvenes mexicanos*
}

Psychometric properties of the Work Preference Inventory (WPI) in a sample of young Mexican adults

Recepción: 09 Junio 2014 | Aprobación: 24 Enero 2017

\author{
Julio C. Penagos-Corzo \\ Universidad de las Américas, México \\ ORCID: http://orcid.org/0000-0002-4879-3033 \\ Mayra E. Olvera Esquivel \\ Universidad de las Américas, México \\ Sheila Pintado Cucarella \\ Universidad de las Américas, México
}

a Autor de correspondencia. Correo electrónico: julioc.penagos@udlap.mx

\section{RESUMEN}

El presente trabajo analiza las propiedades psicométricas del Work Preference Inventory (WPI; Amabile, Hill, Hennessey \& Tighe, 1994) traducido al español, en una muestra de 762 participantes mexicanos de entre 17 y 27 años. La consistencia interna del instrumento indica un alfa de Cronbach general de 0.72 , así como de 0.71 para la escala de motivación intrínseca y de 0.69 para la de motivación extrínseca. Se realizaron análisis factoriales para agrupar los reactivos en dos escalas primarias y cuatro secundarias. Además, se hizo la baremación a partir de la muestra estudiada. Los resultados indican que el WPI es un instrumento válido y confiable para medir la motivación en poblaciones universitarias.

\section{Palabras clave}

estructura factorial; motivación extrínseca; motivación intrínseca; validación psicométrica; WPI

Para citar este artículo: Penagos-Corzo, J., Olvera, M., \& Pintado, S. (2017). Propiedades psicométricas del Work Preference Inventory (WPI) en una muestra de adultos jóvenes mexicanos. Universitas Psychologica 16(2), 1-15. https://doi.org/10.11144/Javeriana.upsy 16-2.ppwp

\begin{abstract}
In the present study was analyzed the psychometric properties of the Work Preference Inventory (WPI; Amabile, Hill, Hennessey \& Tighe, 1994) translated into Spanish, in a sample of 762 Mexican participants between 17 and 27 years old. The internal consistency of the instrument indicates a general Cronbach alpha of 0.72 , as well as 0.71 and 0.69 for intrinsic and extrinsic motivation scales, respectively. Factor analyzes were performed to pool the items in two primary and four secondary scales. In addition, the norms were developed from the study sample. The results indicate that the WPI is a valid and reliable instrument to measure motivation in college populations.

Keywords

factorial structure; extrinsic motivation; intrinsic motivation; psychometric validation; WPI
\end{abstract}




\section{Introducción}

La motivación es un componente fundamental de la psicología ya que es el núcleo de la regulación biológica, cognitiva y social (Ryan \& Deci, 2000). A lo largo del tiempo, se han propuesto diferentes maneras de explicar y definir la motivación en función de las diversas perspectivas. Desde una perspectiva fisiológica, las conductas motivadas involucran comportamiento de desplazamiento, aumento del alertamiento, suelen ser anticipatorias y surgir como resultado de relojes biológicos o de otros estímulos internos o externos (Escobar \& Aguilar, 2002). A la vez, bajo esta óptica innumerables procesos neurobiológicos están implicados, dentro de los que destacan los circuitos de recompensa cerebral (Navratilova $\&$ Porreca, 2014). Mientras que desde la teoría psicodinámica se concibe a la pulsión como la fuerza motivadora del individuo (Ciacciulli, Mazza \& Soto, 2013; Pine, 2005). Desde un punto de vista conductista, la motivación puede estudiarse como un impulso derivado de operaciones medio-ambientales que genera una reacción conductual del individuo (Aló \& Cançado, 2013). Por último, desde la postura fenomenológica se relaciona con la satisfacción de necesidades tanto físicas como psicológicas, las cuales incluyen a la auto-realización, el crecimiento personal y la expresión creativa (McElroy, 2013).

La motivación, entendida como la fuerza o fuerzas que señalan la dirección, energía y persistencia del desempeño comportamental, puede simplificarse en intrínseca y extrínseca (Cerasoli, Nicklin \& Ford, 2014). La motivación extrínseca se define como aquellas fuerzas que generan una actividad en orden de obtener una recompensa extrínseca o cumplir una demanda (fuerza externa); mientras que la motivación intrínseca es definida como la energía innata con la que cuentan los organismos para ser efectivos al tratar con su medio (fuerza interna o autodeterminación) (Deci \& Ryan, 1985; Swanson \& Whittinghill, 2015). De Charms (1983) señala que el punto decisivo de la diferencia entre motivación intrínseca y extrínseca recae en el sentimiento o percepción personal de causa y efecto. Es así como la satisfacción se experimenta en una actividad, que es percibida como intrínsecamente motivadora, al cubrir la necesidad personal que tiene el individuo de sentirse la causa y el efecto de sus acciones.

Las aproximaciones a la motivación como una variable dicotómica (intrínseca-extrínseca) o como un continuum con dos polos, ha permitido relacionarla con otras variables para observar si uno de los polos tiene efectos diferenciales en contraposición con el otro polo. En general se ha reportado que la motivación intrínseca tiene una relación positiva con la tenacidad, la creatividad y el bienestar, entre otras variables de tipo deseable (Deci \& Ryan, 1985; Ryan \& Deci, 2000). Aunque también existe evidencia de ventajas positivas de la motivación extrínseca, por ejemplo en la rehabilitación neurocognitiva (Holland et al., 2016). Se ha reportado que eventos relacionados con la motivación extrínseca pueden tener efectos en la motivación intrínseca; por ejemplo, Boniecki y Moore (2003) señalan que las recompensas y castigos disminuyen la motivación intrínseca. Por otro lado, esta puede aumentar si la recompensa es retenida (Amabile, 1983, 1985, 1996; Amabile, Hill, Hennessey \& Tighe, 1994; Henessey, 2003; Runco, 2007).

Por otra parte, un estudio meta-analítico reciente reporta que los incentivos y la recompensa no son incompatibles; de hecho se encuentran datos que señalan que si los incentivos se relacionan directamente con el rendimiento, la motivación intrínseca no es un buen predictor de este (Cerasoli et al., 2014). Sin embargo, el mismo trabajo en cuestión destaca que la motivación intrínseca resulta más esencial en la calidad del desempeño, mientras que la motivación extrínseca impacta más sobre la cantidad de este.

De cualquier forma, la motivación es altamente valorada por lo que produce (Ryan \& Deci, 2000) y si bien pueden verse sus efectos en una gran cantidad de variables y ámbitos, en el mundo cotidiano es importante observar sus implicaciones prácticas en la 
vida diaria, académica, laboral y hasta en la salud. En este sentido, se ha estudiado la motivación intrínseca y su relación con la salud, tanto física como psicológica, y se han encontrado resultados que relacionan la motivación intrínseca con una mayor adherencia al tratamiento, un mayor bienestar psicológico, un mayor número de conductas saludables y una mayor autonomía personal (Deci \& Ryan, 2012; Edmunds, Ntoumanis \& Duda, 2007; Fortier, Sweet, O’Sullivan \& Williams, 2007; Halvari, Halvari, Bjørnebekk, \& Deci, 2010; Hagger, Chatzisarantis \& Harris, 2006; Niemiec, Halvari, Bjørnebekk \& Deci, 2009; Simoneau \& Bergeron, 2003; Swanson \& Whittinghill, 2015).

En el ámbito escolar, se ha relacionado la motivación intrínseca con un mayor rendimiento académico (Barkoukis, Taylor, Chanal, \& Ntoumanis, 2014; Guay et al., 2010; Spada \& Moneta, 2014; Wentzel, Battle, Russell \& Looney, 2010), así como un mejor autoconcepto de sí mismo en relación a los estudios (Chatzistamatiou, Dermitzaki, Efklides $\&$ Leondari, 2015), una mayor persistencia y conducta prosocial (Froiland, Oros, Smith \& Hirchert , 2012), una mayor autonomía (Jang, Reeve \& Deci, 2010; Katz, Kaplan \& Gueta, 2010) y un mayor bienestar psicológico (Deci \& Ryan, 2000; Froiland et al., 2012; Furrer \& Skinner, 2003; Hudson, 2014).

Por otro lado, en la vida laboral la participación de la motivación intrínseca vs. motivación extrínseca también resulta relevante (Dysvik \& Kuvaas, 2011), no solo en el trabajo mismo, sino en la recuperación después de una jornada extenuante de trabajo (Ten Brummelhuis \& Trougakos, 2014). Además, se ha observado que en el caso de pago por desempeño, los incentivos pueden tener un efecto positivo, aun si este es un desempeño creativo (Zhang \& Long, 2013). La relación entre motivación y creatividad tiene relativa evidencia (Liu, et al., 2013; Peng, Cherng, Chen \& Lin, 2013; Stuhlfau, 2010). En términos de qué tipo de motivación es más conveniente para esta variable, no hay datos concluyentes. Si bien se ha reportado que la motivación intrínseca favorece la creatividad (de Jesus, Rus, Lens \&
Imaginário, 2013; Hon \& Kim, 2007), también existen datos en un sentido opuesto. Por ejemplo, se reporta que la motivación extrínseca tiene efectos positivos (Burroughs, Dahl, Moreau, Chattopadhyay \& Gorn, 2011) o que las recompensas pueden aumentar los beneficios de la motivación intrínseca (Grant \& Berry, 2011). También se ha propuesto y encontrado evidencia que indica que el proceso creativo funciona bajo un modelo recíproco de motivación entre la orientación a uno y la orientación a los otros (Forgeard \& Mecklenburg, 2013). Aunque en ámbitos laborales hay evidencia que señala que los efectos de los incentivos serán dependientes de los tipos de estructura motivacional (Sohn, Ottmann \& Schöffski, 2011). De la misma forma, los efectos positivos de la motivación intrínseca estarán asociados al tipo de trabajo desempeñado (Park \& Rainey, 2012).

Debido a las relaciones de la motivación intrínseca y extrínseca con diferentes variables psicológicas como las anteriormente señaladas, resulta particularmente útil contar con un instrumento que permita evaluarla. En este sentido, existen diversas aproximaciones. Por ejemplo, la Sport Motivation Scale (SMS) (Martens \& Webber, 2002) parte de un modelo que incorpora las características intrínsecas y extrínsecas de la motivación. Dicha escala, adaptada a diferentes poblaciones es frecuentemente utilizada para evaluar aspectos motivacionales en el ámbito deportivo (Balaguer, Castillo \& Duda, 2007; GraneroGallegos, Baena-Extremera, Gómez-López, Sánchez-Fuentes \& Abraldes, 2014; GraneroGallegos, Gómez-López, Extremera, Abraldes \& Rodríguez-Suárez, 2012; Teo et al., 2015) a la vez que han sido cuestionadas sus propiedades psicométricas (Pelletier, Rocchi, Vallerand, Deci \& Ryan, 2013). En otros ámbitos, por ejemplo en la sexualidad, también se han construido instrumentos para medir la motivación extrínseca - intrínseca (MEI). Un ejemplo de ello es la Sexual Motivation Scale (SexMS) (Gravel, Pelletier \& Reissing, 2016). A la par, otros dominios, como la religiosidad, igualmente se han abordado en la construcción de instrumentos desde la óptica de la motivación 
aquí comentada. En este sentido, destaca la Christian Religious Internalization Scale, que también cuenta adaptaciones psicométricas a diversas poblaciones (Brambilla, Manzi \& Regalia, 2014; De Oliveira, 2005). Por otro lado, para evaluar la motivación emprendedora, se desarrolló una escala que como las anteriores mide la MEI, además de otras dimensiones tales como la seguridad y la independencia (BoadaGrau, Sánchez-García, Viardot, Boada-Cuerva \& Vigil-Colet, 2016). La MEI a es ampliamente estudiada en la vida escolar, de ahí que también existan medidas para este ámbito. Un ejemplo es la Escala de Motivación Educativa (EME) (Núñez, Martín-Albo \& Navarro, 2005; Núñez, Martín-Albo, Navarro \& Grijalvo, 2006). En un sentido más amplio y con más énfasis en uno de los polos del continuum de motivación intrínseca - extrínseca, el Intrinsic Motivation Inventory (IMI) fue desarrollado con base en la teoría de la auto-determinación (McAuley, Duncan \& Tammen, 1989; Ryan, 1982). Este instrumento se ha adaptado también para diversas poblaciones (Eow Yee, Wan Zah bte Wan, Roselan \& Rosnaini, 2010; Gutiérrez, 2014) y es utilizado en diferente ámbitos tales como en la investigación de la esquizofrenia (Choi, Mogami \& Medali, 2010), del aprendizaje de habilidades motoras (Grand et al., 2015) o del rendimiento académico en relación con diferencias de género (Cortright, Lujan, Blumberg, Cox \& DiCarlo, 2013). Por su uso amplio es importante destacar la Motivation Assessment Scale (MAS) (Conroy, Fox, Bucklin \& Good, 1996; Durand \& Crimmins, 1988; Kearney, Cook, Chapman \& Bensaheb, 2006; Koritsas \& Iacono, 2013; Shogren \& Rojahn, 2003) o por sus aproximaciones estadísticas alternativas el Assessment of Individual Motivation (AIM) (Stark, 2011), o bien por su amplitud el Individual Directions Inventory (IDI) (Kabacoff, 2008). Finalmente, en relación a instrumentos que midan la motivación en el trabajo, pocos parecen abordarla desde un enfoque de motivación extrínca - intrínseca. Uno de ellos es la The Work Extrinsic and Intrinsic Motivation Scale (WEIMS) (Tremblay, Blanchard, Taylor, Pelletier \& Villeneuve, 2009).
Los instrumentos antes citados, aunque usados en varios escenarios, no fueron creados explícitamente para poblaciones frecuentemente estudiadas, como universitarios o adultos trabajadores. En este sentido, el Work Preference Inventory (WPI), es un instrumento que mide la motivación intrínseca y extrínseca dirigida a estudiantes universitarios y a adultos trabajadores (Amabile et al., 1994). Las escalas fueron creadas con base en el trabajo de Deci y Ryan (1985). Los elementos considerados en la medición de motivación del WPI en relación a la motivación intrínseca son la autodeterminación (preferencia por elección y autonomía), competencia (orientación y preferencia por los retos), involucramiento con la tarea, curiosidad (preferencia por la complejidad), interés (disfrute y alegría). Por otra parte, los elementos relacionados con la motivación extrínseca son los concernientes a la evaluación, referentes al reconocimiento y a la competencia, interés en el dinero o en incentivos tangibles y concentración en lo que dicen los demás (Amabile et al., 1994).

A la fecha, el WPI ha sido usado en estudios que vinculan la motivación intrínseca-extrínseca con aspectos de la personalidad (Kangas, 2002; Katz, 2002), la resiliencia (Ghasem \& Hosseinchari, 2012), con la motivación en contextos competitivos (Abuhamdeh \& Csikszentmihalyi, 2009), con la creatividad (Stuhlfaut, 2010; Ailisha \& Zhang, 2006), con variables relacionadas al desempeño académico y vida escolar (Brownlow, Gilbert \& Reasinger, 1997; Moneta \& Siu, 2002), así como a la vida laboral (Kenan-Smalls, 2011; Plant, \& Ren, 2010), entre otras variables.

Aunque las propiedades psicométricas del WPI han sido confirmadas en estudios posteriores (Loo, 2001; Stuhlfaut, 2010) al trabajo de Amabile et al. (1994), no existen adaptaciones del instrumento al español, como tampoco parecen haber baremos o normas para poblaciones específicas. Por tanto el presente estudio tiene como objetivo realizar la adaptación al español del WPI, analizar sus propiedades psicométricas, así como desarrollar las normas 
del WPI, para una población mexicana de universitarios jóvenes.

\section{Método}

\section{Participantes}

Se seleccionaron por disponibilidad a 762 estudiantes de dos universidades privadas del centro de México. Del total de la muestra, un $57,3 \%$ eran mujeres $(\mathrm{N}=437)$ y un $42,7 \%$ hombres $(\mathrm{N}=325)$ con un rango de edad de 17 a 27 años.

\section{Instrumentos}

Se utilizó el Work Preference Inventory (Amabile et al., 1994), diseñado para evaluar la motivación extrínseca e intrínseca de estudiantes universitarios y trabajadores adultos hacia sus actividades de trabajo. Consta de 30 reactivos con cuatro opciones de respuesta: "Nunca o casi nunca es cierto en mi caso", "Algunas veces es cierto en mi caso", "Frecuentemente es cierto en mi caso", "Siempre o casi siempre es cierto en mi caso".

El WPI, o Inventario de Preferencias Laborales, consta de dos escalas primarias de orientación intrínseca y extrínseca de 15 reactivos cada una y cuatro sub-escalas. La escala Intrínseca primaria (alfa de Cronbach= 0.79), está subdivida en las escalas secundarias de Desafío y de Gozo, las cuales presentan una consistencia interna de 0.74 y 0.71 , respectivamente. La subescala Desafío se compone de cinco reactivos y la escala Gozo de 10. La Escala Extrínseca primaria (alfa de Cronbach $=0.78$ ), se subdivide en las escalas secundarias de Extraversión, con 10 reactivos y Compensación, con cinco reactivos. Tienen una consistencia interna de 0.71 y 0.78 , respectivamente.

En primer lugar, el instrumento fue traducido del inglés por cuatro traductores profesionales. Las traducciones fueron consensuadas por los miembros del equipo de investigación y se procedió a la traducción inversa. Nuevamente los traductores profesionales revisaron e hicieron modificaciones. Finalmente una versión al español fue enviada al equipo de trabajó que consensuó la versión final, la cual fue probada en un estudio piloto.

\section{Procedimiento}

El instrumento fue administrado individualmente a los 762 participantes después de que firmaban un consentimiento informado. Para determinar la capacidad discriminatoria de los reactivos que conforman el instrumento traducido, este se aplicó a 200 estudiantes. Posteriormente el instrumento fue aplicado a los 562 participantes restantes. Los participantes fueron invitados a participar durante sus sesiones de clase para responder individualmente al término de estas si así lo decidían.

\section{Resultados}

Análisis de la capacidad discriminatoria de los reactivos

El análisis de capacidad de discriminación de los 30 reactivos traducidos al español, realizado mediante la prueba $t$ de Student, muestra que 21 reactivos presentan una significancia $<0.001$, salvo los reactivos 15,22 y 30 que presentan una significancia $<0.05$. Los reactivos $1,2,8,9$, 14 y 23 no presentan capacidad discriminatoria. La numeración de los reactivos corresponde a la numeración de Amabile et al. (1994).

\section{Análisis de componentes principales}

Se realizó un análisis factorial exploratorio por medio de componentes principales con rotación varimax. Este primer análisis arrojó siete factores que en conjunto explicaban el $53.82 \%$ de la varianza. Sin embargo, para permitir la congruencia con el instrumento original se redujeron los factores en el análisis. Así pues, resultaron dos factores; el Factor 1 mostró un 
autovalor (eigenvalue) de 3.142 y una varianza explicada del $13.09 \%$. Por otra parte, el Factor 2 mostró un autovalor de 2.907 y una varianza explicada del $12.11 \%$. En conjunto los dos factores explican el 25.2\% de la varianza.

La distribución de los reactivos en los dos factores se determinó por la carga factorial arrojada en la matriz de tipo varimax (ver Tabla 1). Se eliminaron los reactivos 7 y 11 por tener una carga factorial $<0.3$.

TABLA 1

Matriz con rotación varimax y peso factorial

\section{Reactivos}

9. Me gusta enfrentar problemas que son completamer nuevos para mí.

20. Disfruto tratar de resolver problemas complejos.

22. Quiero averiguar qué tan bueno puedo ser en mi trabajo.

24. Lo que más me importa es disfrutar lo que hago.

21. Para mí es importante tener una salida para mi auto expresión

1. Mientras más difícil sea el problema, más disfruto e intentar resolverlo

5. Prefiero resolver las cosas por mi mismo.

15. Para mí, es importante hacer lo que más me gusta.

12. Me siento más tranquilo cuando puedo fijar mis propias metas.

3. Quiero que mi trabajo me proporcione oportunidade para aumentar mis conocimientos y habilidades.

7. La curiosidad es la fuerza motivante detrás de mucl de lo que hago.

2. Estoy profundamente consciente de las metas que debo proponerme para obtener buenas calificacione

18. Me siento fuertemente motivado por el reconocimiento que pueda obtener de los demás.

23. Deseo que otra gente averigüe qué tan bueno realmente puedo llegar a ser en el trabajo

Fuente: elaboración propia.

Debido a la congruencia con el instrumento original, el factor 1 fue renombrado como Escala Primaria de Motivación Intrínseca (MI), mientras que el factor 2 se renombró como Escala Primaria de Motivación Extrínseca (ME). Con base en la obtención de dos las escalas primarias, se realizó un análisis posterior para determinar la viabilidad de posibles subescalas. El análisis factorial de la escala primaria MI indica la existencia de dos factores: El primer factor explica el $19.22 \%$ de la varianza y tiene un autovalor de 2.307. El segundo factor, con un autovalor de 2.291 explica el 19.09\% de la varianza. Los dos factores en su conjunto explican el $38.31 \%$ de la varianza. Así, los reactivos de la escala primaria MI quedaron agrupados en dos subescalas de acuerdo al peso factorial de cada reactivo $(\geq 30)$. Estas subescalas se denominaron Subescala con Orientación de Gozo (SOG) y Subescala con Orientación de Desafío (SOD) al tomar como referencia las escalas secundarias propuestas por Amabile et al., (1994) (ver tabla 2).

TABLA 2

Matriz con rotación varimax. Estructura factorial de la Escala Primaria MI

\begin{tabular}{llc}
\hline \multirow{2}{*}{ Reactivos } & \multicolumn{2}{c}{ Factores } \\
\cline { 2 - 3 } & Goz0 & Desafio \\
\hline R24 & 0.60 & \\
R22 & 0.59 & \\
R15 & 0.58 & \\
R2 & 0.57 & \\
R3 & 0.55 & \\
R12 & 0.54 & \\
R21 & 0.40 & \\
R20 & & 0.84 \\
R1 & & 0.76 \\
R9 & & 0.75 \\
R5 & & 0.38 \\
R7 & & 0.30 \\
\hline
\end{tabular}

Fuente: elaboración propia.

De la misma manera que en el análisis anterior, se procedió a realizar un análisis factorial, en este caso de la Escala Primaria ME. El análisis señala dos factores, uno con un autovalor de 2.442 y una explicación de la varianza de $24.42 \%$, y un segundo factor con un autovalor de 1.625 , con una explicación del $16.25 \%$ de la varianza. En conjunto los dos factores explican el $40.67 \%$ de la varianza. Los reactivos de la escala primaria $\mathrm{ME}$, se agruparon en las dos 
subescalas de acuerdo al del peso factorial $\geq 30$. Estas subescalas se denominaron Subescala con Orientación de Extraversión (SOE) y Subescala con Orientación de Compensación (SOC) (ver tabla 3).

\section{TABLA 3}

Matriz con rotación varimax. Estructura factorial de la Escala Primaria ME

\begin{tabular}{lll}
\hline \multirow{2}{*}{ Reactivos } & \multicolumn{2}{c}{ Factores } \\
\cline { 2 - 3 } & Extraversión & Compensación \\
\hline R18 & 0.71 & \\
R19 & 0.67 & \\
R13 & 0.66 & \\
R23 & 0.53 & \\
R4 & 0.49 & \\
R10 & 0.49 & \\
R8 & 0.46 & \\
R16 & 0.30 & \\
R6 & & 0.83 \\
R14 & & 0.82 \\
\hline
\end{tabular}

Fuente: elaboración propia.

Análisis de consistencia interna

El análisis de consistencia interna del instrumento, indica un alfa de Cronbach $=0.72$ para el instrumento completo. La escala primaria MI obtuvo un alfa de Cronbach $=0.71$, mientras que para la escala primaria $\mathrm{ME}$, el valor alfa de Cronbach fue de 0.69. En relación las escalas secundarias de MI, tanto la escala SOG como la SOD obtuvieron un alfa de Cronbach de 0.65. En relación a las escalas secundarias, $\mathrm{SOE}$ obtuvo un alfa de Cronbach de 0.68, y SOC de 0.63.

\section{Datos normativos}

Los valores crudos de cada escala fueron convertidos a valores $T$, para poder establecer los baremos. En la tabla 4 se presentan, a manera de perfil las puntuaciones naturales y su conversión a T. Estas puntuaciones se derivan de los valores obtenidos en las escalas primarias MI ( $=38.62$, $\mathrm{DE}=4.44)$ y $\mathrm{ME}(=26.6, \mathrm{DE}=4.79)$, así como de las subescalas SOG $(=24.34, \mathrm{DE}=2.7)$, SOD $(=14.93, \mathrm{DE}=2.32), \mathrm{SOE}(=20.46, \mathrm{DE}=$ $4.19)$ y $\operatorname{SOC}(=6.14, \mathrm{DE}=1.5)$.

\section{TABLA 4}

Puntuaciones naturales y su conversión a puntuaciones $T$

\begin{tabular}{|c|c|c|c|}
\hline $\mathrm{T}$ & $\begin{array}{c}\text { Escala } \\
\text { MI }\end{array}$ & $\begin{array}{c}\text { Escala } \\
\text { ME }\end{array}$ & $\begin{array}{c}\text { Subescala } \\
\text { G }\end{array}$ \\
\hline 78 & & 40 & \\
\hline 77 & & & \\
\hline 76 & & 39 & \\
\hline 75 & & & \\
\hline 74 & & 38 & \\
\hline 73 & & & \\
\hline 72 & & 37 & \\
\hline 71 & & & \\
\hline 70 & & 36 & \\
\hline 69 & & & \\
\hline 68 & & 35 & \\
\hline 67 & & & \\
\hline 66 & & & \\
\hline 65 & & 34 & \\
\hline 64 & & & 28 \\
\hline 63 & & 33 & \\
\hline 62 & 44 & & \\
\hline 61 & & 32 & \\
\hline 60 & 43 & & 27 \\
\hline 59 & & 31 & \\
\hline 58 & 42 & & \\
\hline 57 & & 30 & \\
\hline 56 & & & 26 \\
\hline 55 & 41 & 29 & \\
\hline 54 & & & \\
\hline 53 & 40 & 28 & \\
\hline 52 & & & 25 \\
\hline 51 & 39 & 27 & \\
\hline 50 & & & \\
\hline 49 & 38 & 49 & 24 \\
\hline 48 & & & \\
\hline 47 & & 25 & \\
\hline 46 & 37 & & \\
\hline
\end{tabular}

\section{Discusión}

Los datos del presente estudio permiten señalar que la adaptación del instrumento para la población mexicana es confiable, además de tener un rango de confiabilidad similar al de otros instrumentos con escalas de motivación (D’Anello, 1998; Gauthier, Senécal \& Guauy, 
Julio C. Penagos-Corzo, Mayra E. Olvera Esquivel, Sheila Pintado Cucarella.

2007; Manassero Más \& Vázquez Alonso, 1998; Martens \& Webbwe, 2002; Moreno et al., 2007; Núñez et al., 2006; Ruiz \& Gutiérrez, 2001). Y más importante, los valores de consistencia interna obtenidos se aproximan a los valores del instrumento original. Las alfas reportadas para el WPI original fueron de 0.75 para MI y 0.71 para ME, mientras que aquí fueron de 0.71 y 0.69 respectivamente. Lo mismo para las subescalas, tanto en la tendencia de reactivos como en los valores alfa.

Del instrumento original fueron eliminados ocho reactivos en total. La eliminación procede de la falta de capacidad de discriminación de los reactivos.

Es probable que esta falta de capacidad de discriminación sea producto del proceso de traducción y retraducción. Este proceso se hizo con base en las normas de confiabilidad y validez; sin embargo, el valor semántico de los reactivos requería de adaptaciones significativas a la cultura que de hacerse hubieran modificado sustancialmente al reactivo. Al no ser adaptado a la cultura, las afirmaciones en cada reactivo por lo tanto perdieron parte de su significado. Por ejemplo, el reactivo 23 puede tener una interpretación sobre el trabajo escolar vs. laboral, o bien el concepto absorbente puede tener un sentido externo en el sentido de demandas del medio y no intrínseco en el sentido de apasionarse con algo. El reactivo 8 que fue traducido como "No importan los resultados de un proyecto, estoy satisfecho si siento que he obtenido una nueva experiencia", pudiera ser interpretado como conformidad, así que aún personas con motivación intrínseca estarían dando una respuesta negativa a dicho reactivo.

Los mismos sesgos pueden ocurrir en reactivos eliminados de la escala de motivación intrínseca. Por ejemplo el reactivo 1 pudo haber sido interpretado como una tendencia a la mediocridad o a un exceso de egoísmo el no interés de los demás. Dicho reactivo afirma "No me preocupa lo que otras personas piensan de mi trabajo". La cultura mexicana es una cultura colectivista en contraste con la cultura anglosajona que es más individualista (Shkodriani, 1995; Smith et al., 2011). Esto matizaría este y otros reactivos que en el instrumento original aparecen como extrínsecos. Estos matices culturales pueden estar igualmente presentes en el reactivo 2 de la versión final en español (4 en el original). En el instrumento en inglés aparece en motivación extrínseca, pero en nuestro caso el análisis factorial lo ubicó en el factor MI. Este reactivo dice "Estoy profundamente consciente de las metas que debo proponerme para obtener buenas calificaciones". Es probable que la sintaxis haga que lo importante de la oración sea la acción del sujeto sobre la conciencia de las metas y el proponerse las mismas, y el efecto sea obtener buenas calificaciones, que sería la parte extrínseca de la oración. Esto además puede vincularse con lo que sugiere Amabile et al. (1994) en torno a que los artistas creativos que tienen una fuerte motivación intrínseca están interesados tanto por el problema creativo como por ganar el reconocimiento de sus iguales y del público.

La reducción de factores también fue similar al proceso que siguió Amabile et al. (1994). Ellos encontraron seis factores iniciales que redujeron a dos, mientras que en nuestro caso fueron siete iniciales, también ajustados a dos. La reducción de factores puede hacerse para ajustar el instrumento a la propuesta teórica (Arruza et al., 2005; Núñez et al., 2006; Vallerand, Pelletier, Blais, Senècal y Vallières, 1992). Esto permitió un acoplamiento con los factores del instrumento original y facilitó el agrupamiento de los reactivos a las dos dimensiones: reactivos enfocados a detectar la Motivación Intrínseca y la Motivación Extrínseca.

El instrumento original no cuenta con normas, ya que solo determina el tipo de motivación y el tipo de orientación dependiendo del promedio obtenido del puntaje por escala y subescala. Por lo que se puede decir que la creación de una escala de perfil mediante puntajes $T$, mejora las características del instrumento. Así, se puede afirmar que estos puntajes estandarizados proporcionaron un marco de referencia para interpretar la calificación que obtuvo cada estudiante y, de esta manera, determinar su perfil motivacional. Estas normas son aplicables 
a poblaciones con las características similares a la muestra.

En el presente trabajo se analizaron las propiedades psicométricas del WPI. Sin embargo, puede recomendarse para futuras investigaciones sobre la evaluación de la motivación, realizar una adaptación o modificación al instrumento, en donde se aumente tanto el número de factores como el de reactivos. En este sentido, quizá pueda usarse como un referente la Escala de motivación, de Decy y Ryan (1985). En segundo lugar, es recomendable especificar las definiciones de los reactivos a la situación académica de los estudiantes universitarios, ya que algunos de los reactivos están basados mayormente en el ámbito laboral. Y por último, es deseable más adelante correlacionar esta escala con otras escalas e instrumentos, validados y estandarizados, que evalúen la motivación, para así contar con una visión más amplia de la validez del instrumento.

La detección de la motivación intrínseca y la motivación extrínseca en estudiantes universitarios resulta relevante, tanto por el impacto que la motivación tiene en diversos ámbitos como incluso en la orientación vocacional. Finalmente, y a manera de conclusión, el presente estudio valida y confirma la consistencia interna de un instrumento para múltiples usos en la psicología de la motivación y por otro lado lo mejora con la generación de normas para la población mexicana con las características de la muestra estudiada.

\section{Referencias}

Abuhamdeh, S., \& Csikszentmihalyi, M. (2009). Intrinsic and Extrinsic Motivational Orientations in the Competitive Context: An Examination of Person-Situation Interactions. Journal of Personality, 77(5), 1615-1635. doi: https://doi.org/10.1111/j.1 467-6494.2009.00594.x

Ailisha, L., \& Zhang, Q. (2006). A Study of Graduate Students' Creative Motivation. Psychological Science, 29(4), 857-860.

Aló, R. R., \& Cançado, C. X. (2013). Coming to terms with motivation in the behavior- analytic literature. Psychological Record, 63(3), 641-654. doi: https://doi.org/10.111 33/j.tpr.2013.63.3.016

Amabile, T. M. (1983). The Social Psychology of Creativity. New York: Springer-Verlag.

Amabile, T. M., \& Brandeis, U. (1985). Motivation and creativity. Effects of motivational orientation on creative writers. Journal of Personality and Social Psychology, 48(2), 393-39.

Amabile, T. M. (1996). Creativity in Context. Boulder, Colorado: Westview press.

Amabile, T. M., Hill, K. G., Hennessey, B. A., \& Tighe, E. M. (1994). The Work Preference Inventory: Assessing intrinsic and extrinsic motivational orientations. Journal of Personality and Social Psychology, 66(5), 950-967. doi: https://doi.org/10.103 7/0022-3514.66.5.950

Arruza, J. A., Arribas, S., González, O., Balagué, G., Romero S., \& Ruiz, L. M. (2005). Desarrollo y validación de una versión preliminar de la Escala de Competencia Emocional en el deporte. European Journal of Human Movement, 23(4), 114-120.

Barkoukis, V., Taylor, I., Chanal, J., \& Ntoumanis, N. (2014). The relation between student motivation and student grades in physical education: A 3year investigation. Scandinavian Journal of Medicine $\mathcal{E}$ Science in Sports, 24(5), 406-414. doi: https://doi.org/10.1111/sms.1 2174

Balaguer, I., Castillo, I., \& Duda, J. L. (2007). Propiedades psicométricas de la escala de motivación deportiva en deportistas Españoles. Revista Mexicana de Psicología, 24(2), 197-207.

Boada-Grau, J., Sánchez-García, J., Viardot, E., Boada-Cuerva, M., \& Vigil-Colet, A. (2016). Anales de Psicología, 32 (2), 571-577. doi: https://doi.org/10.6018/analesps.32.2.2 08381

Boniecki, K. A., \& Moore, S. (2003). Breaking the Silence: Using a Token Economy to Reinforce Classroom Participation. Teaching of Psychology, 30(3), 224-231. 
Brambilla, M., Manzi, C., \& Regalia, C. (2014). Italian validation of the Christian Religious Internalization Scale. TPMTesting, Psychometrics, Methodology in Applied Psychology, 21(2), 135-148.

Brownlow, S., Gilbert, N. M., \& Reasinger, R. D. (1997). Motivation, personality preferences and interests in college students. Journal of Psychological Practice, 3(4), 128-140.

Burroughs, J., Dahl, D., Moreau, C., Chattopadhyay, A., \& Gorn, G. (2011). Facilitating and rewarding creativity during new product development. Journal of Marketing, 75(4), 53-67. doi: https://doi.org $/ 10.1509 / \mathrm{jmkg} .75 .4 .53$

Cerasoli, C. P., Nicklin, J. M., \& Ford, M. T. (2014). Intrinsic Motivation and Extrinsic Incentives Jointly Predict Performance: A 40-Year Meta-Analysis. Psychological Bulletin, 140(4), 980-1008. doi: https://doi. org/10.1037/a0035661

Chatzistamatiou, M., Dermitzaki, I., Efklides, A., \& Leondari, A. (2015). Motivational and affective determinants of selfregulatory strategy use in elementary school mathematics. Educational Psychology, 35 (7), 835-850. doi: https://doi.org/10.1080/0144 3410.2013 .822960

Choi, J., Mogami, T., \& Medalia, A. (2010). Intrinsic motivation inventory: An adapted measure for schizophrenia research. Schizophrenia Bulletin, 36(5), 966-976. doi: https://doi.org/10.1093/schbu $1 / \mathrm{sbp030}$

Ciacciulli, S. M., Mazza, A., \& Soto, R. (2013). Abordaje histórico del constructo motivación. Un estudio focalizado en las necesidades actuales del campo educativo. Acta Académica, 87-90. Recuperado de htt p://www.aacademica.org/000-054/415

Conroy, M. A., Fox, J. J., Bucklin, A., \& Good, W. (1996). An analysis of the reliability and stability of the Motivation Assessment Scale in assessing the challenging behaviors of persons with developmental disabilities. Education E3 Training in Mental Retardation $\mathbb{E}$ Developmental Disabilities, 31(3), 243-250.
Cortright, R. N., Lujan, H. L., Blumberg, A. J., Cox, J. H., \& DiCarlo, S. E. (2013). Higher levels of intrinsic motivation are related to higher levels of class performance for male but not female students. Advances in Physiology Education, 37(3), 227-232.

D'Anello, S. (1998). Motivación hacia la salud: Conceptualización y medición. Revista de la facultad de medicina, Universidad de Los Andes. 7(1), 1-4.

De Charms, R. (1983). Personal Causation: The internal affective determinants of behavior. New York: Academic Press.

De Jesus, S., Rus, C., Lens, W., \& Imaginário, S. (2013). Intrinsic motivation and creativity related to product: A meta-analysis of the studies published between 1990-2010. Creativity Research Journal, 25(1), 80-84. doi: https://doi.org/10.1080/10400419.201 3.752235

De Oliveira, J. B. (2005). Motivação intrínseca/ extrínseca da vivência religiosa: Uma escala revisitada. Psicologia Educação Cultura, 9(2), 453-475.

Deci, E. \& Ryan (1985). Intrinsic motivation and self-determination in human behavior. New York: Plenum Press.

Deci, E., \& Ryan, R. (2000). The "what" and "why" of goal pursuits: Human needs and the self-determination of behavior. Psychological Inquiry, 11(4), 227-268.

Deci, E. \& Ryan, R. (2012). Selfdetermination theory in health care and its relations to motivational interviewing: a few comments. International Journal of Behavioral Nutrition \&8 Physical Activity, 9(1), 24-29. doi: https://doi.org/10.1186/14 79-5868-9-24

Durand, V. M., \& Crimmins, D. B. (1988). Identifying the variables maintaining selfinjurious behavior. Journal of Autism and Developmental Disorders, 18(1), 99-117.

Dysvik, A., \& Kuvaas, B. (2011). Intrinsic motivation as a moderator on the relationship between perceived job autonomy and work performance. European Journal of Work and Organizational 
Psychology, 20(3), 367-387. doi: https://doi. org/10.1080/13594321003590630

Edmunds, J., Ntoumanis, N., \& Duda, J. (2007). Adherence and well-being in overweight and obese patients referred to an exercise on prescription scheme: A selfdetermination theory perspective. Psychology of Sport And Exercise, 8(5), 722-740. doi: https://doi.org/10.1016/j.psyc hsport.2006.07.006

Eow Yee, L., Wan Zah bte Wan, A., Roselan, B., \& Rosnaini, M. (2010). Stability of the Intrinsic Motivation Inventory (IMI) For the Use of Malaysian Form One Students in ICT Literacy Class. Eurasia Journal of Mathematics, Science $\mathbb{E}$ Technology Education, 6(3), 215-226.

Escobar, C., \& Aguilar, R. (2002). ¿Qué son las conductas motivadas? Una breve historia. En C. Escobar \& R. A. Aguilar. Motivación y conducta. Sus bases biológicas. (pp. 1-12). México, D. F: Manual Moderno.

Forgeard, M. C., \& Mecklenburg, A. C. (2013). The two dimensions of motivation and a reciprocal model of the creative process. Review of General Psychology, 17(3), 255-266. doi: https://doi.org/10.1037/a003 2104

Fortier, M., Sweet, S., O'Sullivan, T., \& Williams, G. (2007). A self-determination process model of physical activity adoption in the context of a randomized controlled trial. Psychology of Sport and Exercise, 8(5), 741-757.

Froiland, J., Oros, E., Smith, L., \& Hirchert, T. (2012). Intrinsic Motivation to Learn: The Nexus between Psychological Health and Academic Success. Contemporary School Psychology, 16(1), 91-100.

Furrer, C., \& Skinner, E. (2003). Sense of relatedness as a factor in children's academic engagement and performance. Journal of Educational Psychology, 95(1), 148-162.

Gauthier, L., Senecal, C. \& Guay, F. (2007). Construction and validation of the Motivation to have a Child Scale (MCS).
Eurepean Review of Applied Psychology. 57(2), 77-89.

Ghasem, M., \& Hosseinchari, M. M. (2012). Psychological resilience and intrinsicextrinsic motivation: The mediating role of self-efficacy. Journal of Iranian Psychologists, 8(33), 61-72.

Grand, K. F., Bruzi, A. T., Dyke, F. B., Godwin, M. M., Leiker, A. M., Thompson, A. G., \& ... Miller, M. W. (2015). Why self-controlled feedback enhances motor learning: Answers from electroencephalography and indices of motivation. Human Movement Science, 43, 23-32. doi: https://doi.org/10.1016/j.humov .2015 .06 .013

Granero-Gallegos, A., Baena-Extremera, A., Gómez-López, M., Sánchez-Fuentes, J. A., \& Abraldes, J. A. (2014). Psychometric Properties of the "Sport Motivation Scale (SMS)" Adapted to Physical Education. Journal of Sports Science EO Medicine, 13(4), 801-807.

Granero-Gallegos, A., Gómez-López, M., Extremera, A. B., Abraldes, J. A., \& Rodríguez-Suárez, N. (2012). La motivación autodeterminada en el balonmano amateur. Revista Iberoamericana de Diagnóstico y Evaluación Psicologica, 33(1), 147-171.

Grant, A. M., \& Berry, J. W. (2011). The necessity of others is the mother of invention: Intrinsic and prosocial motivations, perspective taking, and creativity. Academy of Management Journal, 54(1), 73-96. doi: https://doi.org/10.5465/ AMJ.2011.59215085

Gravel, E. E., Pelletier, L. G., \& Reissing, E. D. (2016). Doing it' for the right reasons: Validation of a measurement of intrinsic motivation, extrinsic motivation, and amotivation for sexual relationships. Personality and Individual Differences, 92, 164-173. doi: https://doi.org/10.1016/j.paid .2015 .12 .015

Guay, F., Chanal, J., Ratelle, C. F., Marsh, H. W., Larose, S., \& Boivin, M. (2010). Intrinsic, identified, and controlled types 
of motivation for school subjects in young elementary school children. British Journal of Educational Psychology, 80(4), 711-735.

Gutiérrez, M. (2014). Relaciones entre el clima motivacional, las experiencias en educación física y la motivación intrínseca de los alumnos. Retos: Nuevas Perspectivas de Educación Física, Deporte y Recreación, 26(junio-diciembre), 9-14.

Hagger, M. S., Chatzisarantis, N. D., \& Harris, J. (2006). From Psychological Need Satisfaction to Intentional Behavior: Testing a Motivational Sequence in Two Behavioral Contexts. Personality and Social Psychology Bulletin, 32(2), 131-148. doi: htt ps://doi.org/10.1177/0146167205279905

Halvari, A., Halvari, H., Bjørnebekk, G., \& Deci, E. L. (2010). Motivation and anxiety for dental treatment: Testing a self-determination theory model of oral self-care behaviour and dental clinic attendance. Motivation EF Emotion, 34(1), 15-33. doi: https://doi.org/10.1007/s11031010-9154-0

Hennessey, B. A. (2003) The Social Psychology of Creativity. Scandinavian Journal of Educational Research. 47(3), 253-261.

Holland, A. A., Hughes, C. W., Harder, L., Silver, C., Bowers, D. C., \& Stavinoha, P. L. (2016). Effect of motivation on academic fluency performance in survivors of pediatric medulloblastoma. Child Neuropsychology, 22(5), 570-586. doi: https://doi.org/10.1080/09297049.201 5.1023272

Hon, A. Y., \& Kim, T. Y. (2007). Work overload and employee creativity: The roles of goal commitment, task feedback from supervisor, and reward for competence. En M. Afzalur Rahim (Ed.) Current Topics in Management, Vol 12 (pp. 193-211). New Brunswick, NJ: Routledge.

Hudson, T. M. (2014). Impact of stress-coping strategies on perceived stress, intrinsic motivation, and self-efficacy levels of students. Education theses, dissertations and projects, paper 36 .
Jang, H., Reeve, J., \& Deci, E. L. (2010). Engaging Students in Learning Activities: It Is Not Autonomy Support or Structure but Autonomy Support and Structure. Journal of Educational Psychology, 102 (3), 588-600.

Kabacoff, R. I. (2008). Issues of global and local norm use in assessments of motivation. International Journal of Testing, 8(4), 367-383. doi: https://doi.org/10.1080/ 15305050802435128

Kangas, B. L. (2002). Intrinsic and extrinsic factors contributing to career development in the dramatic arts. Dissertation Abstracts International: Section B: The Sciences and Engineering, 63(6-B), 3056.

Katz, H. S. (2002). The relationship of intrinsic motivation, cognitive style, and tolerate of ambiguity, and creativity in scientists. Dissertation Abstracts International: Section B: The Sciences and Engineering, 62(11-B), 5419.

Katz, I., Kaplan, A., \& Gueta, G. (2010). Students' Needs, Teachers' Support, and Motivation for Doing Homework: A CrossSectional Study. Journal of Experimental Education, 78(2), 246-267.

Kearney, C. A., Cook, L. C., Chapman, G., \& Bensaheb, A. (2006). Exploratory and Confirmatory Factor Analyses of the Motivation Assessment Scale and Resident Choice Assessment Scale. Journal of Developmental and Physical Disabilities, 18(1), 1-11. doi: https://doi.org/10.1007/s1 0882-006-9000-1

Kenan-Smalls, Y. (2011). Diversity and inclusion in information technology from an age perspective: Motivating and managing information technology professionals across multiple generations in the workforce. Dissertation Abstracts International Section A, 72,1366

Koritsas, S., \& Iacono, T. (2013). Psychometric comparison of the Motivation Assessment Scale (MAS) and the Questions About Behavioral Function (QABF). Journal of Intellectual Disability Research, 57(8), 747-757. doi: https://doi.org/10.1111/ jir. 12022 
Liu, G., Zhang, S., Zhang, J., Lee, C., Wang, Y., \& Brownell, M. (2013). Autonomous motivation and Chinese adolescents' creative thinking: The moderating role of parental involvement. Creativity Research Journal, 25(4), 446-456. doi: https://doi.org $/ 10.1080 / 10400419.2013 .843401$

Loo, R. (2001). Motivational orientations toward work: an evaluation of the work preference inventory (student form). Measurement $\mathscr{E}$ Evaluation in Counseling 83 Development, 33(4), 222-231.

Manassero Más, M. \& Vázquez Alonso, A. (1998). Validación de una escala de motivación de logro. Psicothema, 10(2), 333-351.

Martens, M. \& Webbwe, S. N. (2002). Psycometric properties of the Sport Motivation Scale: An evaluation with collage varsity athletes from the U.S. Journal of Sport and Exercise Psychology. 24(3), 254-270.

McAuley, E., Duncan, T., \& Tammen, V. V. (1989). Psychometric properties of the Intrinsic Motivation Inventory in a competitive sport setting: A confirmatory factor analysis. Research Quaterly for Exercise and Sports, 60(1), 48-58.

McElroy, R. (2013). Higher Education: Motivation to Teach. Global Education Journal, 1(1), 45-49.

Moneta, G. B., \& Siu, C. M. Y. (2002). Trait intrinsic and extrinsic motivations, academic performance, and creativity in Hong Kong College. Journal of College Student Development, 43(5), 664-83.

Moreno, J. A., López, G., Martínez, G., Alonso C. M., \& González-Cutre, D., (2007). Validación preliminar de la escala de percepción del clima motivacional de los iguales (CMI) y la escala de meta en el ejercicio (GOES), con practicantes españoles de actividad físico-deportivas. Revista Iberoaméricana de Psicología del Ejercicio y el Deporte, 1(2), 13-28.

Niemiec, C., Halvari, H., Bjørnebekk, G., \& Deci G. (2009). Aspiring to physical health: The role of aspirations for physical health in facilitating long-term tobacco abstinence. Patient Education and Counseling, 74(2), 250-257.

Navratilova, E., \& Porreca, F. (2014). Reward and motivation in pain and pain relief. Nature Neuroscience, 17(10), 1304-1312. doi: https://doi.org/10.1038/nn.3811 .

Nu\#n\#ez, J. L., Marti\#n-Albo, J., \& Navarro, J. G. (2005). Validacio\#n de la versio\#n espan\# ola de la E\#chelle de Motivation en E\#ducation. Psicothema, 17(2), 344-349.

Núñez, J. 1., Martín-Albo, J., Navarro, J. G., \& Grijalvo, F. (2006), Validación de la Escala de Motivación Educativa (EME) en Paraguay. Revista Interamericana De Psicología / Interamerican Journal of Psychology, 40(3), 391-398.

Park, S., \& Rainey, H. G. (2012). Work motivation and social communication among public managers. The International Journal of Human Resource Management, 23(13), 2630-2660. doi: https://doi.org/10. 1080/09585192.2011.637060

Pelletier, L. G., Rocchi, M. A., Vallerand, R. J., Deci, E. L., \& Ryan, R. M. (2013). Validation of the revised sport motivation scale (SMS-II). Psychology of Sport and Exercise, 14(3), 329-341. doi: https://doi.or g/10.1016/j.psychsport.2012.12.002

Peng, S., Cherng, B., Chen, H., \& Lin, Y. (2013). A model of contextual and personal motivations in creativity: How do the classroom goal structures influence creativity via self-determination motivations?. Thinking Skills and Creativity, 10, 50-67. doi: https://doi.org/10.1016/j.tsc .2013 .06 .004

Pine, F. (2005). Theories of Motivation in Psychoanalysis. En E. S. Person, A. M. Cooper, G. O. Gabbard, E. S. Person, A. M. Cooper, G. O. Gabbard (Eds.), The American psychiatric publishing textbook of psychoanalysis (pp. 3-19). Arlington, VA: American Psychiatric Publishing, Inc.

Plant, R., \& Ren, J. (2010). A comparative study of motivation and entrepreneurial intentionality: Chinese and American perspectives. Journal of Developmental 
Entrepreneurship, 15(2), 187-204. doi: https ://doi.org/10.1142/S1084946710001506.

Ruiz, L. Graupera, J. L., \& Gutiérrez, M. (2001). Adaptación, validación y análisis psicométrico del test AMPLET de motivación de logro en educación física de T. Nishida, a población escolar española. Revista de psicología del deporte y control motor. 353(7), 210-219.

Runco, A. M. (2007). Creativity. Theories and team: Research development and practice. London: Academic Press.

Ryan, R. M. (1982). Control and information in the intrapersonal sphere: An extension of cognitive evaluation theory. Journal of Personality and Social Psychology, 43(3), 450-461.

Ryan, R. M., \& Deci, E. L. (2000). Selfdetermination theory and the facilitation of intrinsic motivation, social development, and well-being. American Psychologist, 55(1), 68-78. doi: https://doi.org/10.1037/0 003-066X.55.1.68

Shkodriani, G. L. (1995). Individualism and Collectivism Among University Students in Mexico and the United States. Journal of Social Psychology, 135(6), 765-772.

Shogren, K. A., \& Rojahn, J. (2003). Convergent reliability and validity of the Questions About Behavioral Function and the Motivation Assessment Scale: A replication study. Journal of Developmental and Physical Disabilities, 15 (4), 367-375. doi: https://doi.org/10.1023/A:1026314316977

Simoneau, H., \& Bergeron, J. (2003). Factors affecting motivation during the first six weeks of treatment. Addictive Behaviors, 28(7), 1219-1241. doi: https://doi.org/10.1 016/S0306-4603(02)00257-5

Smith, P. B., Torres, C. V., Hecker, J., Chua, C., Chudzikova, A., Degirmencioglu, S., \& ... Yanchuk, V. (2011). Individualismcollectivism and business context as predictors of behaviors in cross-national work settings: Incidence and outcomes. International Journal of Intercultural Relations, 35(4), 440-451. doi: https://doi.o $\mathrm{rg} / 10.1016 /$ j.ijintrel.2011.02.001
Sohn, S., Ottmann, S., \& Schöffski, O. (2011). Intrinsic motivational drivers in medical networks from a neuroscientific perspective. Journal of Neuroscience, Psychology, And Economics, 4(3), 192-204. doi: https://doi.org/10.1037/a0025017

Spada, M. M., \& Moneta, G. B. (2014). Metacognitive and motivational predictors of surface approach to studying and academic examination performance. Educational Psychology, 34(4), 512-523. doi: https://doi.org/10.1080/01443410.201 3.814196

Stark, S., Chernyshenko, O. S., Drasgow, F., Lee, W. C., White, L. A., \& Young, M. C. (2011). Optimizing prediction of attrition with the U.S. Army's Assessment of Individual Motivation (AIM). Military Psychology, 23(2), 180-201. doi: https:// doi.org/10.1080/08995605.2011.550234

Stuhlfaut, M. W. (2010). Evaluating the Work Preference Inventory and its Measurement of Motivation in Creative Advertising Professionals. Journal of Current Issues $\mathbb{E}$ Research In Advertising (CTC Press), 32(1), 81-93.

Swanson, L. R., \& Whittinghill, D. M. (2015). Intrinsic or extrinsic? Using videogames to motivate stroke survivors: A systematic review. Games For Health, 4(3), 253-258.

Ten Brummelhuis, L. L., \& Trougakos, J. P. (2014). The recovery potential of intrinsically versus extrinsically motivated off\#job activities. Journal of Occupational and Organizational Psychology, 87(1), 177-199.

Teo, E., Khoo, S., Wong, R., Wee, E., Lim, B., \& Rengasamy, S. S. (2015). Intrinsic and extrinsic motivation among adolescent ten-pin bowlers in kuala lumpur, malaysia. Journal of Human Kinetics, 7(45), 241-251. doi: https://doi.org/10.1515/hukin-2015-00 25

Tremblay, M. A., Blanchard, C. M., Taylor, S., Pelletier, L. G., \& Villeneuve, M. (2009). Work Extrinsic and Intrinsic Motivation Scale: Its value for organizational psychology research. Canadian Journal of 
Behavioural Science/Revue Canadienne Des Sciences Du Comportement, 41 (4), 213-226. doi: https://doi.org/10.1037/a0015167

Vallerand, R. J., Pelletier, L.G., Blais, M. R., Brière, N. M., Senècal, C., \& Vallières, E. F. (1992). The Academic Motivation Scale: A measure of intrínsic, extrinsic and amotivation in education. Educational and Psychological Measurement. 52(3), 1003-10017

Wentzel, K. R., Battle, A., Russell, S. L., \& Looney, L. B. (2010). Social supports from teachers and peers as predictors of academic and social motivation. Contemporary Educational Psychology, 35(3), 193-202.

Zhang, Y., \& Long, L. (2013). The impact of pay for performance on employees' creativity: Moderating effect of person-job fit and mediating effect of creative self-efficacy. Acta Psychologica Sinica, 45(3), 363-376.

\section{Notas}

* Artículo de investigación 